\title{
Generating Isoform-Specific Antibodies: Lessons from Nucleocytoplasmic Glycoprotein Skp1
}

\author{
Christopher M. West, Hanke van der Wel, Zoiesha Chinoy, \\ Geert-Jan Boons, Ted J. Gauthier, Carol M. Taylor, and Yuechi Xu
}

\section{Contents}

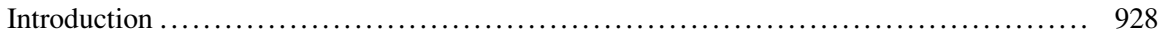

Strategies to Develop Isoform-Specific Abs .................................... 929

Murine Monoclonal Abs ................................................. 930

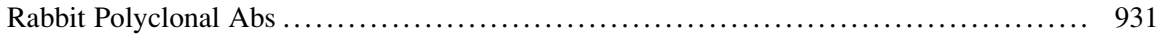

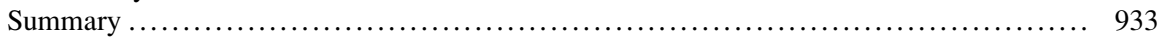

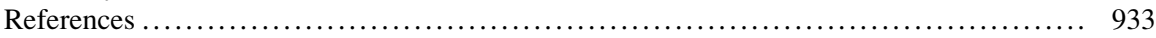

\begin{abstract}
Antibodies that discriminate protein isoforms differing by modifications at specific amino acids have revolutionized studies of their functions. Skp1 is a novel nucleocytoplasmic glycoprotein that is hydroxylated at proline-143 and then O-glycosylated by a pentasaccharide attached via a GlcNAc $\alpha 1,4$ (trans)hydroxyproline linkage. Skp1 isoform-specific antibodies were successfully
\end{abstract}

C.M. West $(\bowtie) \cdot$ H. van der Wel $\bullet$ Y. Xu

Department of Biochemistry and Molecular Biology, Oklahoma Center for Medical Glycobiology, Health Sciences Center, University of Oklahoma, Oklahoma City, OK, USA

e-mail: Cwest2@ouhsc.edu; hanke-van-der-wel@ouhsc.edu; yuechi-xu@ouhsc.edu

Z. Chinoy $\bullet$ G.-J. Boons

Department of Chemistry and Complex Carbohydrate Research Center, University of Georgia, Athens, GA, USA

e-mail: zchinoy1@uga.edu; gjboons@ccrc.uga.edu

T.J. Gauthier

AgCenter Biotechnology Laboratory, LSU AgCenter, Baton Rouge, LA, USA

e-mail: tgauthier@agcenter.lsu.edu

C.M. Taylor

Department of Chemistry, Louisiana State University, Baton Rouge, LA, USA

e-mail: cmtaylor@1su.edu 
obtained by immunizing mice or rabbits with KLH-coupled synthetic peptides bearing either unmodified Pro, 4(trans)-hydroxyproline, or D-GlcNAc 1,4 (trans)-hydroxyproline, and screening with corresponding BSA-conjugates or by Western blotting toward a panel of Skp1 isoforms. Antibodies specific for Skp1 or HO-Skp1 were not found in exhaustive murine trials, yet monospecific polyclonal antibodies were readily achieved in rabbits without crossadsorption. In all cases, antibodies were specific at the protein but not the peptide level, which suggests that conformation comprises part of the basis for recognition and which should be considered when developing screening strategies.

\title{
Keywords
}

Monoclonal antibody $\bullet$ Polyclonal antibody $\bullet$ Skp1 $\bullet$ Cytoplasmic glycosylation • Isoform-specific antibody - Hydroxyproline - Ubiquitin ligase • Oxygen • Synthetic glycopeptide $\bullet$ Conformational epitope

\author{
Abbreviations \\ BSA Bovine serum albumin \\ CRL Cullin-Ring ligase \\ FBP F-box protein \\ KLH Keyhole limpet hemocyanin \\ $\mathrm{mAb}$ Monoclonal antibody \\ $\mathrm{pAb}$ Polyclonal antibody \\ SCF Ub-ligase complex consisting of Skp1, cullin-1, an FBP, and Rbx1 \\ $\mathrm{Ub}$ Ubiquitin
}

\section{Introduction}

Skp1 is an adaptor linking F-box proteins with cullin-1 in the highly conserved SCF class of E3 ubiquitin (Ub) ligases (Willems et al. 2004). The E3 ${ }^{\mathrm{SCF}}$ Ub ligases, also known as CRL1, mediate the K48-polyubiquitination of a broad range of centrally important regulators of cell cycling, physiology, and differentiation. Their specificity is controlled by the choice of F-box protein (FBP) selected from the pool of dozens to hundreds that are genomically encoded in a given organism, and often by posttranslational modification, such as phosphorylation, of the target protein. CRL1, as well as most of the other known CRLs (7 in humans), are regulated via the cullin scaffold protein by activating neddylation and inhibitory binding of Cand1 (Deshaies and Joazeiro 2009).

In protists such as the social amoeba Dictyostelium (West et al. 2010) and the apicomplexan human pathogen Toxoplasma gondii (Xu et al. 2012a), a novel posttranslational modification of the Skp1 subunit may represent an additional level of CRL1-specific control (Wang et al. 2011; Sheikh et al. 2014). The modification is initiated by 4-hydroxylation of Pro143, followed by step-wise addition of five sugars (Fig. 1), leading to the accumulation at steady state of a remarkably homogenous pentasaccharide. Evidence indicates that $\mathrm{O}_{2}$ availability 


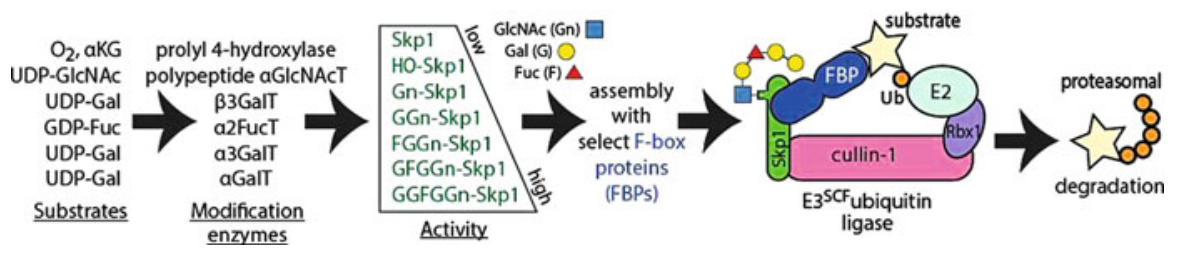

Fig. 1 Proposed role of Skp1 modification in regulation of protist proteomes. Availability of enzyme substrates listed in the left column regulates the activity of modification enzymes in the second column, which sequentially generate the Skp1 isoforms shown in the third column. Increasing modification promotes interaction with select F-box proteins, potentially leading to increased $\mathrm{E} 3{ }^{\mathrm{SCF}} \mathrm{Ub}$-ligase activity and degradation of target substrates. $\alpha \mathrm{KG}=\alpha$-ketoglutarate or 2-oxoglutarate (The $\mathrm{E}^{\mathrm{SCF}} \mathrm{Ub}$-ligase schematic was originally published in Schafer et al. (2014), (C) the American Society for Biochemistry and Molecular Biology)

is rate limiting for Skp1 hydroxylation via a prolyl 4-hydroxylase, PhyA (Xu et al. 2012b), and genetic studies indicate that PhyA (West et al. 2007) is an ortholog of the human $\mathrm{O}_{2}$-sensor that activates the transcriptional cofactor hypoxia-inducible factor- $\alpha$ for polyubiquitination by CRL2 and subsequent proteasomal degradation (Kaelin and Ratcliffe 2008). Dictyostelium may regulate its proteome in response to $\mathrm{O}_{2}$-availability via selective proteolysis involving Skp1 modifications (Fig. 1), compared to the mammalian mechanism of transcriptional activation.

Isoform-specific Abs that differentiate discrete modification states of Skp1 have proved useful for monitoring the hydroxylation and glycosylation status of Skp1 in enzymatic assays (van der Wel et al. 2011) and in cells during hypoxia (Xu et al. 2012a) or when glycosyltransferase genes are under- or overexpressed (Zhang et al. 2012; Schafer et al. 2014). Development of Abs that are specific for hydroxylation and glycosylation modifications remains highly empirical (Ingale et al. 2007; Fukuda 2012), and here factors that have contributed to the success of current isoform-specific Abs are reviewed.

\section{Strategies to Develop Isoform-Specific Abs}

Despite the apparent novelty of the Skp1 glycan, immunization of mice with native glycosylated Skp1 failed to yield glycan-dependent mAbs (Kozarov et al. 1995). In addition, reactive lectins have not been found. The core trisaccharide is equivalent to the blood group $\mathrm{H}$ type I structure, but is not recognized by a commercial mAb (Abcam ab3355) which reacts with this trisaccharide in other contexts (Yu et al. 2012). Thus, synthetic hydroxypeptides and glycopeptides representing early steps of hydroxylation and GlcNAc addition were used to focus the immune response to the site of modification. Multiple immunization and screening strategies are summarized below for their effectiveness in generating Abs specific for Skp1 (unmodified), HO-Skp1, or GlcNAc-Skp1. 


\section{Murine Monoclonal Abs}

The first strategy was a traditional approach of immunizing mice with 13-mer peptides conjugated via an N-terminal Cys to KLH, using a combination of Freund's complete and incomplete adjuvants. mAbs were screened using an ELISA-type assay with corresponding BSA-peptides for mAbs specific for the target peptide relative to the other two peptides (Wang et al. 2009, 2011). Initial sera were positive with high titers but not specific. High affinity mAbs reactive toward target peptides appeared with good frequency but were equally reactive with all three peptides, suggesting that the desired specificity was not achieved. The majority of these mAbs reacted similarly with all six Skp1 isoforms analyzed based on standard Western blotting of Skp1 isoforms present in cytosolic extracts of mutant strains that accumulated the individual isoforms.

GlcNAc-Skp1 (Gn-Skp1)-specific mAbs were, however, detected by Western blot screening of peptide-reactive hybridoma supernatants derived from mice immunized with the GlcNAc-peptide, at a frequency of approximately one per standard fusion. An example (mAb 1C9) is shown in Fig. 2c, and a second mAb, 2F8, exhibits similar specificity. Both mAbs reacted similarly in ELISA assays toward all three BSA-peptides. Thus, the unique epitope required the presence of additional parts of Skp1 not present in the immunogen. One possibility is that the full-length protein imposes conformational restrictions on the peptide, which is further constrained by the chemical modification, and that the unique epitope(s) is not comprised only of the chemical modification per se.

In contrast, this strategy failed to recover mAbs specific for unmodified Skp1 or HO-Skp1. Screening of peptide-reactive mAbs with full-length Skp1 in the Western blot assay yielded $\mathrm{mAb} 4 \mathrm{H} 2$ that discriminated unmodified Skp1 relative to Gn-Skp1 (Wang et al. 2011). However, mAb 4H2 also bound HO-Skp1 and showed weak reactivity with other isoforms (Fig. 2b). As observed for the above mAbs, $\mathrm{mAb} 4 \mathrm{H} 2$ was not discriminatory at the peptide level. This was the only Skp1 selective $\mathrm{Ab}$ isolated from two fusions.

mAbs that discriminated HO-Skp1 from unmodified Skp1 were also not forthcoming from this approach, despite screening of four fusions from mice immunized with the Hyp-peptide using various adjuvants and cell culture media. Interestingly, several mAbs (3A9, 8G5, 12B6) showed a novel pattern of reactivity at the protein level toward Skp1, HO-Skp1, and GGn-Skp1, with weak reactivity toward FGGnSkp1 and little to no reactivity with the other isoforms including Gn-Skp1 (Fig. 2a). These mAbs were also not specific at the peptide level. The occurrence of the epitope on GGn-Skp1, recognized by a mAb from a mouse that did not encounter this structure, suggests that it represents, at least in part, a novel conformational epitope that is indirectly influenced by the chemical structure, rather than a unique chemical determinant per se, as inferred above for the other mAbs. This may be related to the changes in global Skp1 conformation after GlcNAc addition that are observed in circular dichroism and small-angle X-ray scattering studies (Sheikh et al. 2014). 
Fig. 2 Reactivity of Skp1 isoform-selective antibodies, based on Western blot analysis of cytosolic extracts of mutant strains (labeled at top) that accumulate the indicated isoforms, labeled at bottom. See Fig. 1 for key. More highly glycosylated isoforms migrate more slowly in the SDS-PAGE gel, as revealed by the pan-specific $\mathrm{mAb} 4 \mathrm{E} 1$. The pAbs in panels $D$ and $E$ were diluted 1:5000 (Panels $C-F$ were originally published in Zhang et al. (2012), (C) the American Society for Biochemistry and Molecular Biology)

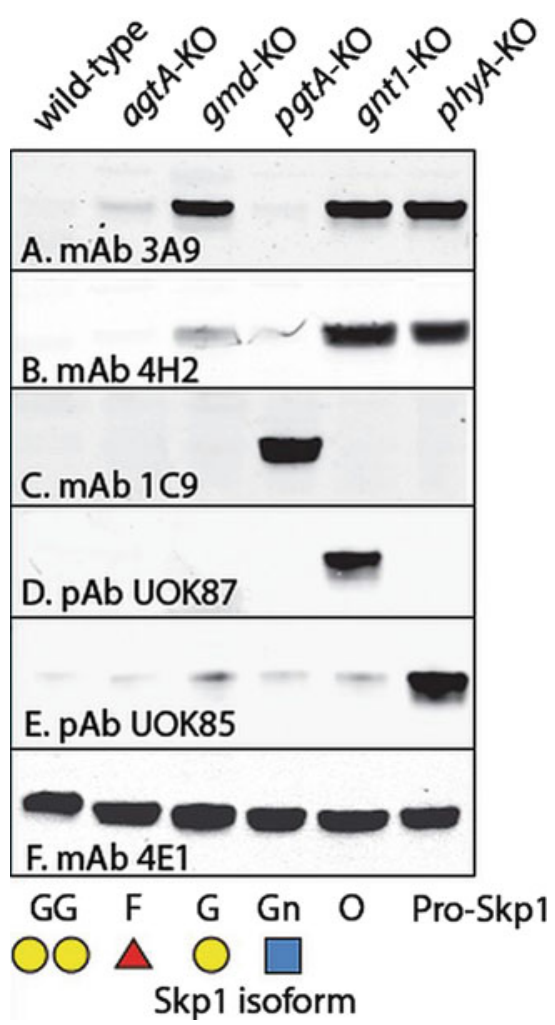

\section{Rabbit Polyclonal Abs}

As a second approach to generate Abs specific for HO-Skp1 or Skp1, rabbits were immunized with the same KLH-peptide conjugates and adjuvant scheme. In this trial, rabbits received final boosts with modified peptides containing a two-amino acid C-terminal extension, with intent to not amplify the immune response toward the unnatural peptide C-terminus. Western blot analysis indicated that one of each the two rabbits exhibited strong preferential reactivity with its corresponding Skp1 isoform (Fig. 2d, e), whereas the other rabbit exhibited strong albeit nonselective reactivity (Zhang et al. 2012). These antisera were also not specific at the peptide level. Thus, rabbits responded strongly and with apparent high frequency to epitopes unique to unmodified or hydroxylated Skp1, outcomes that were not achieved by exhaustive screening of six mouse fusions. The antisera from animals that preferentially responded to Skp1 (UOK87) and HO-Skp1 (UOK85) exhibited high affinity, maintained selectivity over multiple bleeds, and were not improved by affinity purification. 


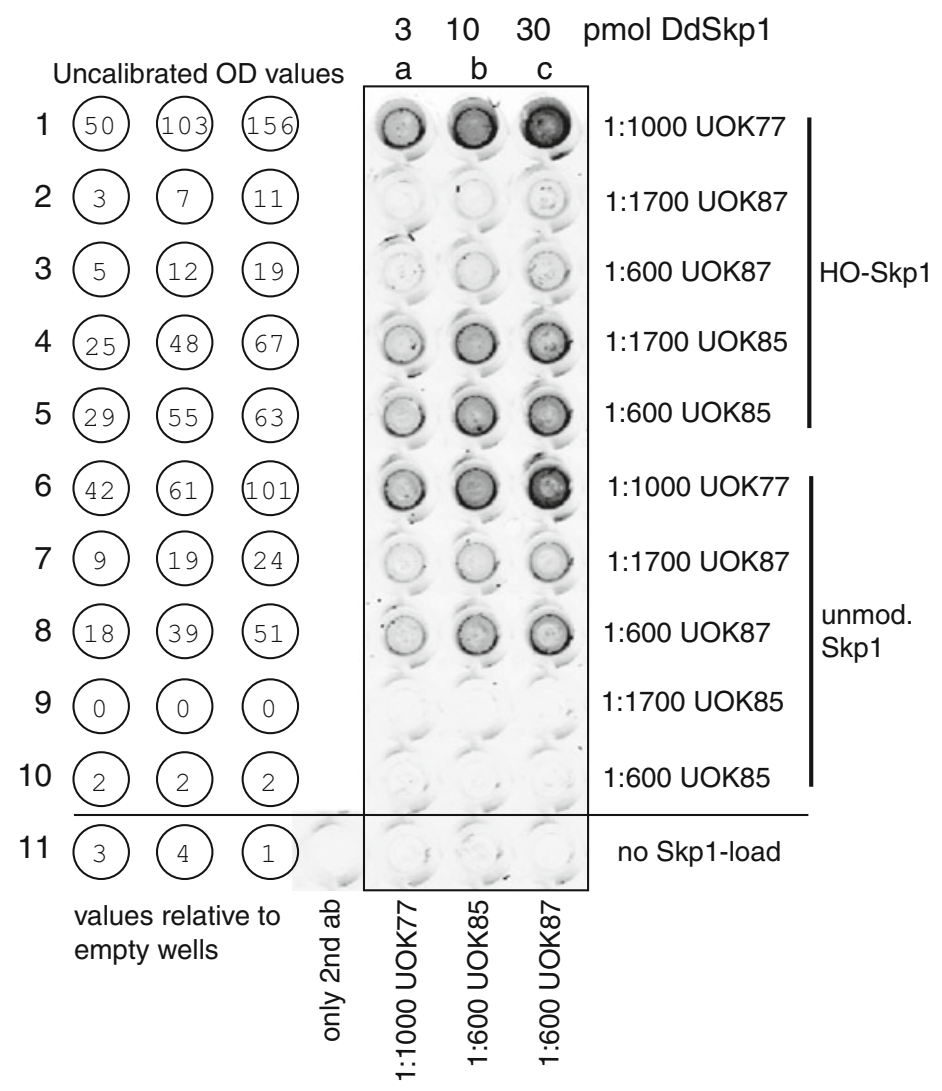

Fig. 3 Fluorescence-based soluble Ab binding assay. The indicated amounts of Skp1 or HO-Skp1 in $50 \mu \mathrm{l}$ were captured for $2 \mathrm{~h}$ in microwells precoated with anti-Skp1 (mAb 3 F9) and blocked with $2 \mathrm{mg} / \mathrm{ml}$ BSA. After washing, the wells were incubated with the indicated dilution of $\mathrm{pAb}$ UOK77 (pan-specific), pAb UOK87, or pAb UOK85 for $2 \mathrm{~h}$, washed, and incubated with 1:10,000 Alexa 680-conjugated goat anti-rabbit IgG in $2 \mathrm{mg} / \mathrm{ml}$ BSA for $2 \mathrm{~h}$. After final washing, wells were imaged in a Li-Cor Odyssey fluorescence scanner (middle panel) and densitometrically quantitated using ImageJ in the left panel. pAb UOK77 confirmed the presence of Skp1 (compare rows 1 \& 6). Row 11, below the line, shows background binding in the absence of bound Skp1

These pAbs were further characterized by sandwich-type solution binding assays that did not involve subjecting Skp1 to denaturing conditions of SDS-PAGE/ Western blotting. Full-length Skp1 isoforms were captured by mAb 3F9 that had been adsorbed to the surface of a 96-well plate and probed with dilution series of the pAbs (Fig. 3). Analysis of pAb UOK85 confirmed exquisite specificity toward HO-Skp1 relative to Skp1 (compare row 4 with 9). pAb UOK87 was found to prefer unmodified Skp1 (compare row 8 with 3), but some reactivity was observed against HO-Skp1 again in agreement with the Western blotting. The results indicate that the epitopes are present on natively folded Skp1. Their presence following denaturing SDS-PAGE (Fig. 2) may result from refolding, as circular dichroism 
studies show that purified Skp1 readily renatures after unfolding (Tan et al. 2008; van der Wel et al. 2011; Sheikh et al. 2014). The higher success rate suggests that rabbits have a broader immune response repertoire toward this class of epitopes.

\section{Summary}

The use of synthetic peptide conjugates successfully focused immune responses to epitopes associated with discrete modification states of Pro143 in full-length Skp1. High affinity pAbs and mAbs were highly selective for unmodified Skp1, HO-Skp1, or GlcNAc-Skp1. Rabbits were especially effective for generating Abs specific for Pro- and Hyp-containing Skp1s, which did not require affinity enrichment or crossadsorption for specificity. Evidence indicates that these epitopes are conformational in nature, requiring screening at the protein level for their detection.

Acknowledgments The research summarized here was supported by NIH grants R01-GM037539 and R01-GM084383 and OCAST grant HR-0141.

\section{References}

Deshaies RJ, Joazeiro CA (2009) RING domain E3 ubiquitin ligases. Annu Rev Biochem 78:399-434

Fukuda MN (2012) Peptide-displaying phage technology in glycobiology. Glycobiology 22:318-325

Ingale S, Wolfert MA, Gaekwad J, Buskas T, Boons GJ (2007) Robust immune responses elicited by a fully synthetic three-component vaccine. Nat Chem Biol 3:663-667

Kaelin WG Jr, Ratcliffe PJ (2008) Oxygen sensing by metazoans: the central role of the HIF hydroxylase pathway. Mol Cell 30:393-402

Kozarov E, van der Wel H, Field M, Gritzali M, Brown RD Jr, West CM (1995) Characterization of FP21, a cytosolic glycoprotein from Dictyostelium. J Biol Chem 270:3022-3030

Schafer CM, Sheikh MO, Zhang D, West CM (2014) Novel regulation of Skp1 by the Dictyostelium AgtA $\alpha$-galactosyltransferase involves the Skp1-binding activity of its WD40repeat domain. J Biol Chem 289:9076-9088

Sheikh MO, Schafer CM, Powell JT, Rodgers KK, Mooers BHM, West CM (2014) Glycosylation of Skp1 affects its conformation and promotes binding to a model F-box protein. Biochemistry 53:1657-1669

Tan A, Tanner JJ, Henzl MT (2008) Energetics of OCP1-OCP2 complex formation. Biophys Chem 134:64-71

van der Wel H, Johnson JM, Xu YC, Karunaratne CV, Wilson KD, Vohra Y, Boons GJ, Taylor CM, Bendiak B, West CM (2011) Requirements for Skp1 processing by cytosolic prolyl 4 (trans)-hydroxylase and $\alpha-\mathrm{N}$-acetylglucosaminyltransferase enzymes involved in $\mathrm{O}_{2}$ signaling in Dictyostelium. Biochemistry 50:1700-1713

Wang ZA, van der Wel H, Vohra Y, Buskas T, Boons GJ, West CM (2009) Role of a cytoplasmic dual-function glycosyltransferase in $\mathrm{O}_{2}$ regulation of development in Dictyostelium. J Biol Chem 284:28896-28904

Wang ZA, Singh D, van der Wel H, West CM (2011) Prolyl hydroxylation- and glycosylationdependent functions of $\mathrm{Skp} 1$ in $\mathrm{O}_{2}$-regulated development of Dictyostelium. Dev Biol 349:283-295 
West CM, van der Wel H, Wang ZA (2007) Prolyl 4-hydroxylase-1 mediates $\mathrm{O}_{2}$ signaling during development of Dictyostelium. Development 134:3349-3358

West CM, Wang ZA, van der Wel H (2010) A cytoplasmic prolyl hydroxylation and glycosylation pathway modifies $\mathrm{Skp} 1$ and regulates $\mathrm{O}_{2}$-dependent development in Dictyostelium. Biochim Biophys Acta 1800:160-171

Willems AR, Schwab M, Tyers M (2004) A hitchhiker's guide to the cullin ubiquitin ligases: SCF and its kin. Biochim Biophys Acta 1695:133-170

Xu Y, Brown KM, Wang ZA, van der Wel H, Teygong C, Zhang DM, Blader IJ, West CM (2012a) The Skp1 protein from Toxoplasma is modified by a cytoplasmic prolyl 4-hydroxylase associated with oxygen sensing in the social amoeba Dictyostelium. J Biol Chem 287:25098-25110

Xu Y, Wang ZA, Green RS, West CM (2012b) Role of the Skp1 prolyl-hydroxylation/ glycosylation pathway in oxygen dependent submerged development of Dictyostelium. BMC Dev Biol 12:31

Yu Y, Mishra S, Song X, Lasanajak Y, Bradley KC, Tappert MM, Air GM, Steinhauer DA, Halder S, Cotmore S, Tattersall P, Agbandje-McKenna M, Cummings RD, Smith DF (2012) Functional glycomic analysis of human milk glycans reveals the presence of virus receptors and embryonic stem cell biomarkers. J Biol Chem 287:44784-44799

Zhang D, van der Wel H, Johnson JM, West CM (2012) The Skp1 prolyl 4-hydroxylase of Dictyostelium contributes glycosylation-independent and -dependent effects on $\mathrm{O}_{2}$-dependent development without affecting Skp1 stability. J Biol Chem 287:2006-2016 